\title{
Effect of treadmill gait training on static and functional balance in children with cerebral palsy: a randomized controlled trial
}

\author{
Luanda A. C. Grecco, Sandra M. Tomita, Thaluanna C. L. Christovão, \\ Hugo Pasini, Luciana M. M. Sampaio, Claudia S. Oliveira
}

\begin{abstract}
Background: Treadmill gait training as a therapeutic resource in the rehabilitation of children with cerebral palsy has recently been the focus of many studies; however, little is still known regarding its effect on static and functional balance in children. Objective: The aim of the present study was to compare the effects of treadmill training and over ground gait training in children with cerebral palsy. Method: A randomized controlled trial with blinded evaluator was conducted with children with cerebral palsy between three and 12 years of age categorized in Levels I to III of the Gross Motor Function Classification System. Assessments were performed before and after the intervention and involved the Berg balance scale as well as the determination of oscillations from the center of pressure in the anteroposterior and mediolateral directions with eyes open and closed. The experimental group was submitted to treadmill training and the control group performed gait training over the ground. The intervention consisted of two 30-minute sessions per week for seven weeks. Results: Both groups exhibited better functional balance after the protocol. The experimental group had higher Berg balance scale scores and exhibited lesser mediolateral oscillation with eyes open in comparison to the control group. Conclusions: Treadmill training had a greater effect on functional balance and mediolateral oscillation in comparison to over ground gait training in children with cerebral palsy. Trial registration: RBR-5v3kg9 (Brazilian Registry of Clinical Trials).
\end{abstract}

Keywords: cerebral palsy; physical therapy; postural balance.

\section{HOW TO CITE THIS ARTICLE}

Grecco LAC, Tomita SM, Christovão TCL, Pasini H, Sampaio LMM, Oliveira CS. Effect of treadmill gait training on static and functional balance in children with cerebral palsy: a randomized controlled trial. Braz J Phys Ther. 2013 Jan-Feb; 17(1):17-23. http://dx.doi.org/10.1590/S1413-35552012005000066

\section{Introduction}

Postural control, specifically postural stability, is a fundamental prerequisite for motor development in children ${ }^{1}$. It is the complex ability of an individual to maintain the center of the gravity of the body over the support base ${ }^{2}$, when an individual is standing still (static balance), in motion (dynamic, functional balance), preparing to perform a movement or preparing to end a movement ${ }^{1}$. Postural control depends on the delicate integration of vision, vestibular and proprioceptive sensations, commands from the central nervous system and neuromuscular responses, particularly muscle strength and reaction time ${ }^{3,4}$. The quantification of displacement of the center of pressure through a stabilometric analysis is often used for the assessment of static balance (stability in a static position) ${ }^{5}$ and the Berg Balance Scale $^{6}$ is used for the assessment of functional balance (postural stability during the performance of functional motor activities).
Deficits in postural control constitute a major limitation to the motor development in children with cerebral palsy $(\mathrm{CP})^{7}$. These children exhibit a number of limitations stemming from postural instability on the performance of static and dynamic tasks, such as sitting, standing and walking ${ }^{7-10}$. The clinical condition of $\mathrm{CP}$ includes neuromuscular deficits, such as the loss of selective motor control and alterations in muscle tone, leading to an imbalance between agonist and antagonist muscles, compromised coordination, sensory alterations and weakness ${ }^{11}$. Postural instability of children with $\mathrm{CP}$ is evident from the greater oscillations of the center of pressure (COP) in the anteroposterior (AP) and mediolateral (ML) directions, even with the use of positioning braces on the lower limbs ${ }^{12}$.

The goal of physical therapy for children with $\mathrm{CP}$ is to promote motor learning through motor and functional training with multiple sensory stimuli. Different approaches have been used to favor selective 
control and the coordination of muscle contrations during gait ${ }^{13-15}$. In recent years, locomotor training on a treadmill has been used in the treatment of children with $\mathrm{CP}$ in an attempt to maximize orthostatism and gait velocity ${ }^{15-21}$. Treadmill training is believed to contribute to postural control by allowing multiple repetitions of the steps of the gait cycle in a rhythmic pattern, thereby improving control between agonist and antagonist muscles and resulting in improved functional and static balance. Previous studies have demonstrated positive results from treadmill training on gait speed and endurance, gross motor function and cardiorespiratory fitness ${ }^{15-21}$. However, these studies do not address the possible effects of treadmill training on postural stability (static and functional balance).

The aim of the present study was to compare the effects of gait training on a moving surface (treadmill) and a fixed surface (ground) on the functional and static balance in functionally ambulatory children with CP.

\section{Method}

The present randomized controlled trial with a blinded evaluator (Figure 1) received approval from the Human Research Ethics Committee of the Universidade Nove de Julho (UNINOVE), São Paulo,
SP, Brazil, under protocol number 409972/2011 and was conducted in accordance to the the ethical norms established in the Resolution 196/96 of the Brazilian National Health Council. All parents/guardians agreed to the participation of their children by signing a statement of informed consent.

\section{Participants}

Participants were recruited and assessed for eligibility using the following inclusion criteria: age between three and 12 years; absence of cognitive or visual impairment that could compromise the performance of the tasks; Levels I, II or III of the Gross Motor Functional Classification System $(\text { GMFCS })^{22}$; and functional ambulation for at least 12 months. Children submitted to orthopedic surgical procedures or neuromuscular block in the 12 months prior to the training sessions and those with orthopedic deformity with indication for surgery were excluded from the study.

\section{Evaluation}

Participants were evaluated prior to and immediately following the gait training protocols. Evaluations included the assessment of functional balance and static balance (stabilometry) as well as a treadmill ramp test protocol.

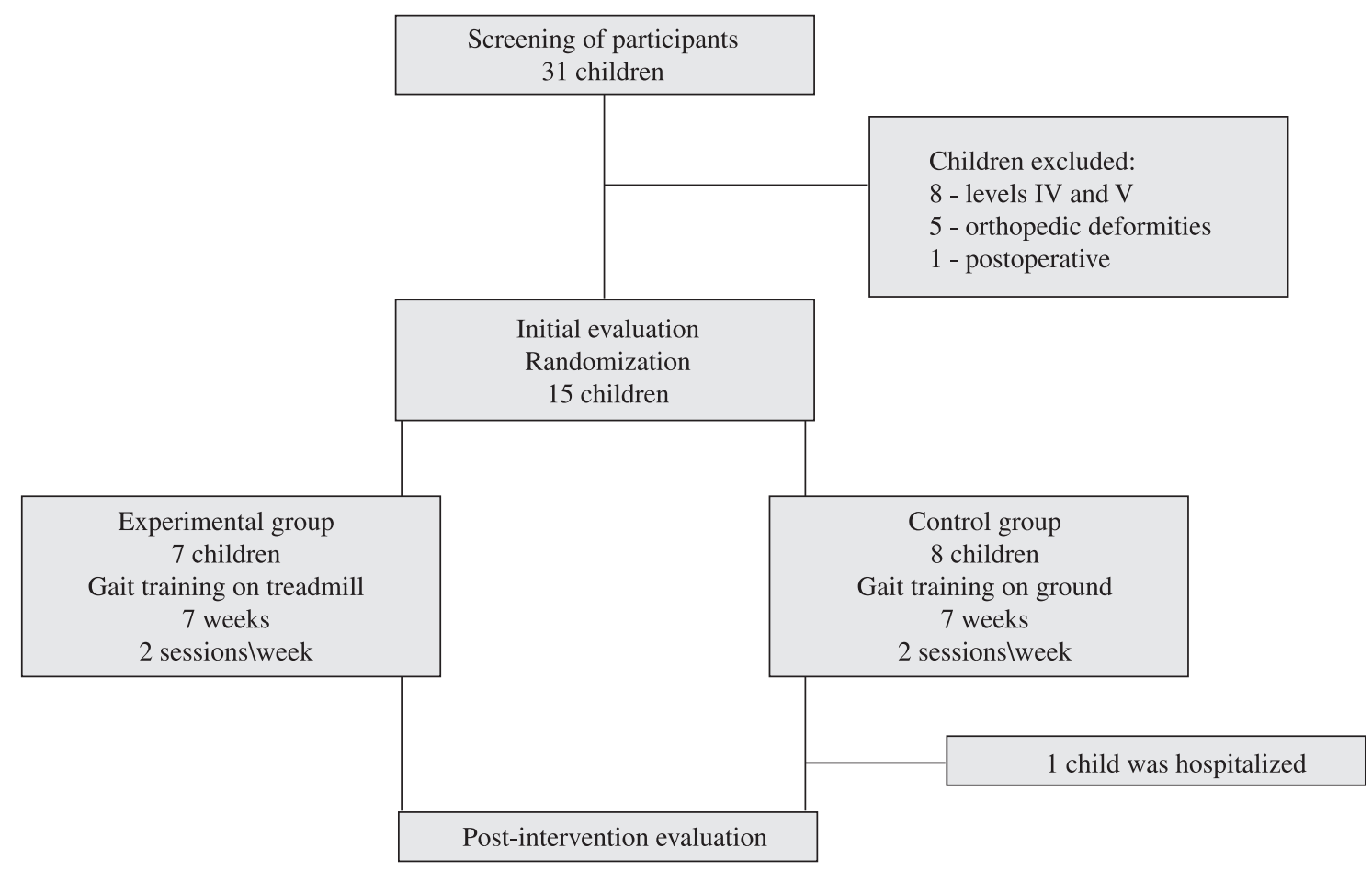

Figure 1. Flowchart of study based on CONSORT. 
Functional balance was assessed using the Berg balance scale, that consists of 14 tasks similar to various activities of daily living. The items are scored on a five-point scale $(0,1,2,3$ or 4$)$, with zero denoting an inability to perform the activity without assistance and four denoting the ability of perform the task with independence. The maximal score is 56 points. The scores are based on the time that a position can be maintained for, the distance that the upper limb is capable to reach in front of the body and the time needed to complete the task. The test was performed with the child clothed and making use of his/her habitual brace and/or gait-assistance device ${ }^{6,23}$.

Static balance was assessed by stabiliometry performed on a Tekscan MatScan ${ }^{\circledR}$ System pressure platform (Boston, USA). This system provides measurements of displacement of the COP along axes $\mathrm{x}(\mathrm{AP})$ and $\mathrm{y}(\mathrm{ML})$. The platform is $5 \mathrm{~mm}$ in thickness, 432 in length and $368 \mathrm{~mm}$ in width and has lines and columns that form an XY grid, with 2288 sensors, resolution of $1.4 \mathrm{sensor} / \mathrm{cm}^{2}$ and sampling frequency of $40 \mathrm{~Hz}$. The child was instructed to remain on the platform in the orthostatic position, barefoot, arms alongside the body or with his/her habitual gaitassistance device, eyes fixed on a point marked at a distance of one meter at the height of the glabella, unrestricted foot base and the heels aligned. The assessment was conducted for 30 seconds under each condition of eyes open (EO) and eyes closed (EC), with the measurements of oscillations of the COP along axes $\mathrm{x}(\mathrm{AP})$ and $\mathrm{y}(\mathrm{ML})^{5}$.

Children were submitted to an exercise test to determine the speed of the treadmill gait training. There are no standardized tests to determine the speed of treadmill gait training for the pediatric population with neurologic disorders. For the present study, a symptom-limited cardiopulmonary effort test was performed on a treadmill (Imbramed Mileniun ATL) with a ramp protocol, beginning at $0.5 \mathrm{~km} / \mathrm{h}$ and increasing the speed by $0.5 \mathrm{~km} / \mathrm{h}$ every minute. The criteria for interrupting the test were: subjective sensation of fatigue, pain in the lower limbs, complex cardiac arrhythmia, sudden increase or drop in blood pressure, increase in heart rate above the maximum predicted for the child's age, intensive shortnessof-breath and drop in oxygenation accompanied by electrocardiographic alteration or signs and symptoms.

The tests were administered on two nonconsecutive days, with the order determined randomly by lots. After fulfilling the eligibility criteria and undergoing the initial evaluation, participants were randomly distributed into an experimental group [gait training on a moving surface (treadmill)] or a control group [gait training on a fixed surface (ground)]. Randomization was performed at a central office using a randomization table. A set of numbered, sealed, opaque envelopes was used to ensure allocation concealment. Each envelop contained a card with the group to which the child was allocated.

\section{Intervention}

Experimental group (treadmill gait training): The treadmill protocol was developed based on recent evidence from studies assessing the effects of treadmill training on children with $\mathrm{CP}^{19,24,25}$. Positive effects have been identified in training protocols involving two or three sessions per week for a period of six to 12 weeks. The protocol employed in the present study was two 30-minute sessions per week over seven consecutive weeks. When necessary, the therapist facilitated the components of the gait cycle during training. The first two sessions were considered a period of familiarization with the treadmill and training was performed with a gradual increase in speed based on the child's degree of tolerance. Sixty to eighty percent maximal tolerance reached on the initial treadmill test was used for the training sessions. Each child walked at $60 \%$ maximal speed in the first and final five minutes of the session and walked at $80 \%$ maximal speed for the other 20 minutes. When applicable, before each session, habitual braces were duly placed by the physiotherapist.

Control group (over ground gait training): Children wore their habitual braces and habitual gait-assistance device, when needed. A prior assessment was performed to determine whether the gait-assistance device was at the adequate height for the child and the necessary adjustments were made. When necessary, therapist facilitated the components of the gait cycle during training. The first two sessions were considered a period of familiarization and the speed and duration of the sessions were self-selected by the children. Over the subsequent six weeks, training sessions lasted 30 minutes. Children were instructed to walk at a comfortable, self-selected pace during the first and final five minutes of the session and encouraged to increase the speed during the other 20 minutes. It was not possible to monitor speed in this group.

Heart rate was monitored to ensure that there was no overload of the cardiovascular system. All participants were instructed to maintain their daily activities and therapy sessions. 
All children were submitted to the aforementioned evaluation procedures immediately following the training protocol.

\section{Statistical analysis}

Data were initially analyzed for Gaussian distribution using the Kolmogorov-Smirnov test. The variables proved to be parametric and were expressed as mean values (95\% confidence intervals). The paired Student's t-test was used for the within group analysis (before and after the gait training protocols and oscillations of COP with EO and EC) and the independent Student's t-test was used for the between group analyses. All $p$-values $<0.05$ were considered significant. Data were organized and tabulated using the Statistical Package for the Social Sciences (SPSS v.19.0).

\section{Results}

Thirty-one children were screened and fifteen were selected for participation in the study. One child in the control group was hospitalized for respiratory problems and therefore did not conclude the study. Table 1 displays the anthropometric characteristics and functional classification of the participants.

The within group analysis demonstrated that both groups achieved higher scores on the Berg balance scale following the gait training protocols. However, the between group analysis demonstrated that the experimental group had statistically better results in comparison to the control group ( $\mathrm{p}=0.01$ ) (Figure 2).

Table 2 displays the results of COP displacement before and after the interventions. No statistically significant differences were detected in the within and between group analyses in the AP direction with

Table 1. Anthropometric characteristics and functional classification of the studied children.

\begin{tabular}{lcc}
\hline & \multicolumn{2}{c}{ Groups } \\
& $\begin{array}{c}\text { Experimental } \\
\mathbf{n}=7\end{array}$ & $\begin{array}{c}\text { Control } \\
\mathbf{n}=7\end{array}$ \\
Gender (female/male)** & $4 / 3$ & $1 / 6$ \\
GMFCS (I/II/III)** & $4 / 1 / 2$ & $3 / 2 / 2$ \\
Age (years)* & $6.8 \pm 2.6$ & $6.0 \pm 1.5$ \\
Body mass $(\mathrm{kg}) *$ & $23.5 \pm 6.5$ & $22.4 \pm 7.0$ \\
Height $(\mathrm{cm}) *$ & $119.0 \pm 13.5$ & $109.5 \pm 4.3$ \\
Body mass index $\left(\mathrm{kg}^{2} / \mathrm{m}\right) *$ & $16.2 \pm 2.9$ & $18.5 \pm 5.5$ \\
\hline
\end{tabular}

GMFCS: Gross Motor Functional Classification System; *data expressed as mean and $95 \%$ confidence interval; **numbers indicate frequency (n) of children in each group. either $\mathrm{EO}$ or $\mathrm{EC}(\mathrm{p}>0.05)$. In the experimental group, six children exhibited less oscillation in the ML direction with $\mathrm{EO}$, with a mean $2.0 \mathrm{~cm}$ less oscillation following treadmill training ( $\mathrm{p}=0.04)$. In the control group, three children exhibited less oscillation in the ML direction with EO, with a mean $0.34 \mathrm{~cm}$ less oscillation following gait training over ground $(\mathrm{p}=0.63)$. No statistically significant differences were found in the within and between group analyses regarding oscillation in the ML direction with EC $(\mathrm{p}>0.05)$.

Displacement of the COP under the conditions of EO and EC was analyzed before and after the gait training protocols. Statistically significant differences were detected in oscillations in the AP direction between the conditions of EO and $\mathrm{EC}$ in both the experimental and control groups ( $\mathrm{p}=0.03$ and $\mathrm{p}=0.01$, respectively). In the within group analyses, no statistically significant differences in oscillation in the AP or ML directions with EC were found before and after the training protocols ( $\mathrm{p}>0.05)$.

\section{Discussion}

Frequent falls constitute one of the most common complaints in children with $\mathrm{CP}$ who have the ability to walk independently. Thus, impaired balance in a functional context is an important issue in the rehabilitation of these children and has been the focus of therapeutic interventions ${ }^{6}$. The present study compared functional and static balance in children with $\mathrm{CP}$ after a protocol of treadmill gait training or a protocol of over ground gait training. Both gait training protocols led to an within group improvement in functional balance. However, treadmill training led to a mean increase of 14.7 points on the Berg balance scale, whereas over ground training led to a 3.2 points

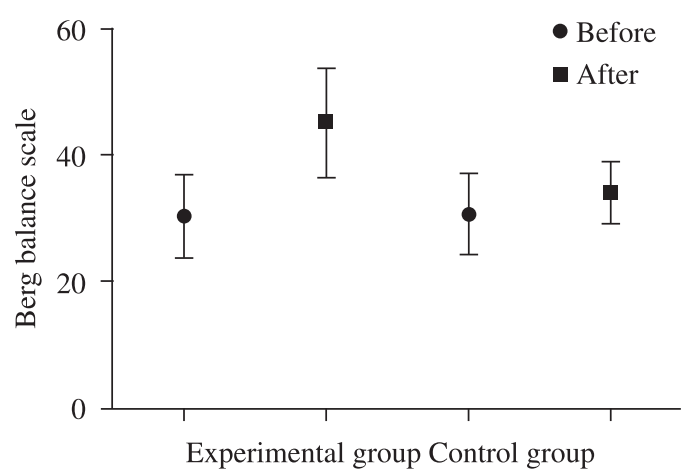

Figure 2. Within and between group analyses of the Berg balance scale before and after the gait training protocols. Mean (95\% confidence intervals) *paired t-test $(\mathrm{p}<0.05)$; **independent $\mathrm{t}$-test $(\mathrm{p}<0.05)$. 
Table 2. Intra-group and inter-group stabilometry analyses before and after gait training protocols.

\begin{tabular}{lcccc}
\hline & \multicolumn{2}{c}{ Experimental group } & \multicolumn{2}{c}{ Control group } \\
& Before & After & Before & After \\
AP oscillation EO $(\mathrm{cm})$ & $1.1(1.0-1.2)$ & $1.0(1.0-1.1)^{*}$ & $1.1(1.0-1.2)$ & $1.0(1.0-1.1)^{*}$ \\
AP oscillation EC $(\mathrm{cm})$ & $1.4(1.1-1.7)$ & $1.3(1.0-1.6)$ & $1.2(1.1-1.2)$ & $1.1(1.1-1.1)$ \\
ML oscillation EO $(\mathrm{cm})$ & $4.2(3.4-5.0)$ & $2.1(0.8-3.5) * / * *$ & $3.6(2.3-5.0)$ & $3.9(3.4-4.4)$ \\
ML oscillation EC $(\mathrm{cm})$ & $5.0(4.5-5.5)$ & $3.5(1.6-5.3)$ & $4.2(2.6-5.9)$ & $4.1(1.6-5.3)$ \\
\hline
\end{tabular}

AP: anteroposterior; ML: mediolateral; EO: eyes open; EC: eyes closed; *paired t-test $(\mathrm{p}<0.05)$; $* *$ independent t-test $(\mathrm{p}<0.05)$.

increase. These findings demonstrate that treadmill training is an important tool on the improvement of functional balance in CP children with GMFCS levels I to III and with independent gait.

Treadmill training allows specific gait training in a rhythmic fashion. Motor tasks require specific muscle control between agonist and antagonist muscles as well as the activation of anti-gravity muscles. Children with $\mathrm{CP}$ exhibit an increase in co-contractions of the proximal and distal muscles, with no coordinated proximal-distal pattern ${ }^{26}$. According to Nashner et al..$^{27}$, CP children present an inverse order of distal and proximal activation and muscle synergies, which compromises responses to disequilibrium. Findings described in the literature demonstrate that treadmill training provides improvements in gait velocity ${ }^{17,19}$, gross motor function ${ }^{15,20}$ and the kinematic pattern of the lower limbs ${ }^{20}$. While no previous study had been conducted to determine the effects of treadmill training on balance, it is suggested that this technique provides an improvement in global coordination and postural control in children, which are fundamental to balance.

Treadmill training activates central pattern generators (CPGs) in the spinal cord at the lumbar level ${ }^{28}$. CPGs are neural activations capable of forming motor patterns, leading to rhythmic, automatic strides, allowing the training of biomechanical components involved in the different phases of gait, postural control and balance ${ }^{14,29}$. The activation of these CPGs and automatic reciprocation mechanisms are an important fundament for the stimulation of gait through treadmill training ${ }^{30}$, as children with $\mathrm{CP}$ employ different postural strategies from those used by children without neurologic impairment.

A pressure platform was used to assess static balance, allowing the quantitative determination of the displacement of the COP in the AP and ML directions. Treadmill training resulted in lesser oscillation in the ML direction with eyes open, whereas no statistically significant differences between groups were found for the AP direction.
The lesser oscillation in the ML direction with eyes open may have stemmed from greater activation of the muscles of the pelvic girdle, thereby allowing greater stability of the pelvis. Smania et al. ${ }^{20}$ found that treadmill training provided improved kinematics of the hips on the sagittal plane, with an increase in the extension of the hips in the support phase of the gait. It should be stressed that a more detailed analysis of muscle activation through electromyography would be required to confirm these hypothesis. However, these evaluations were not performed in this study and thus represent a limitation of this study.

Displacement of the COP was also analyzed with regard to eyes open (EO) and eyes closed (EC). Differences in AP oscillation were found with EO and EC after the gait training protocols. This finding was likely due to the lesser AP oscillation with EO found after the interventions in both groups. No effect of the gait training protocols was found on the displacement of the COP (AP and ML oscillation) with visual restriction. This result is likely due to the important role vision plays in postural control, especially in individuals with neurologic impairment and proprioceptive alterations. However, in contrast to findings described in a previous study with the specific aim to analyze postural oscillations in children with $\mathrm{CP}^{5}$, the present sample did not demonstrate different COP displacement between EO and EC conditions at baseline. Considering the number of participants in the present study, this finding should be studied further.

Indeed the number of participants constitutes an important limitation of the present study. The study population was a convenience sample (children with $\mathrm{CP}$ registered with the Physical Therapy clinic of the UNINOVE, Brazil) and not based on a specific sample size calculation. Moreover, future studies should involve the stratification of the sample during the randomization in regards to the functional levels of the children (GMFCS I/II and GMFCS III). Thus, the results may be considered preliminary 
findings and further studies with a larger number of participants are needed.

\section{Conclusion}

Both gait training on a moving surface (treadmill) and stable surface (ground) resulted in improved functional and static balance. However, treadmill training resulted in a greater functional balance improvement and less mediolateral oscillation with eyes open in children with cerebral palsy.

\section{Acknowledgements}

The financial support from Conselho Nacional de Desenvolvimento Científico e Tecnológico (CNPq) and Coordenação de Aperfeiçoamento de Pessoal de Nível Superior (CAPES).

\section{References}

1. De Kegel A, Dhooge I, Peersman W, Rijckaert J, Baetens T, Cambier D, et al. Construct validity of the assessment of balance in children who are developing typically and in children with hearing impairments. Phys Ther. 2010;90(12):1783-94. PMid:21030662.

2. Overstall P. The use of balance training in elderly people with falls. Rev Clin Gerontol. 2003;13(02):153-61. http:// dx.doi.org/10.1017/S0959259803013273

3. Swanenburg J, De Bruin ED, Favero K, Uebelhart $\mathrm{D}$, Mulder T. The reliability of postural balance measures in single and dual tasking in elderly fallers and non-fallers. BMC Musculoskelet Disord. 2008;9(1):162. PMCid:PMC2614424. http:// dx.doi.org/10.1186/1471-2474-9-162

4. Nashner LM, Black FO, Wall C 3rd. Adaptation to altered support and visual conditions during stance: patients with vestibular deficits. J Neurosci. 2007;2(5):536-44. PMid:6978930.

5. Nobre A, Monteiro FF, Golin MO, Biasotto-Gonzalez D, Corrêa JC, Oliveira CS. Analysis of postural oscillation in children with cerebral palsy. Electromyogr Clin Neurophysiol. 2010;50(5):239-44. PMid:20718335.

6. Kembhavi G, Darrah J, Magill-Evans J, Loomis J. Using the Berg Balance Scale to distinguish balance abilities in children with cerebral palsy. Pediatr Phys Ther. 2002;14(2):92-9. PMid:17053689.

7. Rose J, Wolff DR, Jones VK, Bloch DA, Oehlert JW, Gamble JG. Postural balance in children with cerebral palsy. Dev Med Child Neurol. 2002;44(1):58-63. PMid:11811652.

8. Kyvelidou A, Harbourne RT, Shostrom VK, Stergiou $\mathrm{N}$. Reliability of center of pressure measures for assessing the development of setting postural in infants with or at risk of cerebral palsy. Arch Phys Med Rehabil. 2010;91(10):1593-601. PMid:20875520
PMCid:PMC2948026. http://dx.doi.org/10.1016/j. apmr.2010.06.027

9. Woollacott MH, Shumway-Cook A. Postural dysfunction during standing and walking in children with cerebral palsy: what are the underlying problems and what new therapies might improve balance? Neural Plast. 2005;12(2-3):211-9. http://dx.doi.org/10.1155/NP.2005.211

10. Berger W, Altenmueller E, Dietz V. Normal and impaired development of children's gait. Hum Neurobiol. 1984;3(3):163-70. PMid:6480437.

11. Shumway-Cook A, Hutchinson S, Kartin D, Price R, Woollacott M. Effect of balance training on recovery of stability in children with cerebral palsy. Dev Med Child Neurol. 2003;45(9):591-602. PMid:12948326. http:// dx.doi.org/10.1111/j.1469-8749.2003.tb00963.x

12. Rha DW, Kim DJ, Park ES. Effect of hinged anklefoot orthoses on standing balance control in children with bilateral spastic cerebral palsy. Yonsei Med J. 2010;51(5):746-52. PMCid:PMC2908864. http://dx.doi. org/10.3349/ymj.2010.51.5.746

13. Chagas PSC, Mancini MC, Barbosa AP, Silva PTG. Analysis of the interventions used for grait promotion in children with cerebral palsy: a systematic review of the literature. Rev Bras Fisioter. 2004;8(2):155-63.

14. Silva MS, Daltrário SMB. Cerebral palsy: functional performance after gait training treadmill. Fisioter Mov. 2008;21(30):109-15.

15. Richards CL, Malouin F, Dumas F, Marcoux S, Lepage C, Menier C. Early and intensive treadmill locomotor training for young children with cerebral palsy: A feasibility study. Pediatric Phys Ther. 1997;9(4):158-65.

16. Cherng RJ, Liu CF, Lau TW, Hong RB. Effect of treadmill training with body weight support on gait and gross motor function in children with spastic cerebral palsy. Am J Phys Med Rehabil. 2007;86(7):548-55. PMid:17581289.

17. Dodd KJ, Foley S. Partial body-weight-supported treadmill training can improve walking in children with cerebral palsy: a clinical controlled trial. Dev Med Child Neurol 2007;49(2):101-5. PMid:17253995. http://dx.doi. org/10.1111/j.1469-8749.2007.00101.x

18. Verschuren O, Ketelaar M, Gorter JW, Helders PJ, Uiterwaal CS, Takken T. Exercise training program in children and adolescents with cerebral palsy: a randomized controlled trial. Arch Pediatr Adolesc Med. 2007;161(11):1075-81. PMid:17984410. http:// dx.doi.org/10.1001/archpedi.161.4.356

19. Willoughby KL, Dodd KJ, Shields N, Foley S. Efficacy of partial body weight-supported treadmill training compared with overground walking practice for children with cerebral palsy: a randomized controlled trial. Arch Phys Med Rehabil. 2010;91(3):333-9. PMid:20298820. http://dx.doi.org/10.1016/j.apmr.2009.10.029

20. Smania N, Bonetti P, Gandolfi M, Cosentino A, Waldner A, Hesse S, et al. Improved gait after repetitive locomotor training in children with cerebral palsy. Am J Phys Med Rehabil. 2011;90(2):137-49. PMid:21217461.

21. Johnston TE, Watson KE, Ross SA, Gates PE, Gaughan JP, Lauer RT, et al. Effects of a supported speed treadmill training exercise program on impairment and function 
for children with cerebral palsy. Dev Med Child Neurol. 2011;53(8):742-50. PMid:21679357. http:// dx.doi.org/10.1111/j.1469-8749.2011.03990.x

22. Palisano R, Rosenbaun P, Walter S, Russel S, Wood E, Galuppi B. Development and reliability of a system to classify gross motor function in children with cerebral palsy. Dev Med Child Neurol. 1997;39(4):214-23. PMid:9183258. http://dx.doi.org/10.1111/j.1469-8749.1997.tb07414

23. Berg J. Measuring balance in the elderly: preliminary development of an instrument. Physiother Can. 1989;41(6):304-11. http://dx.doi.org/10.3138/ ptc. 41.6 .304

24. Damiano DL, DeJong SL. A systematic review of the effectiveness of treadmill training and body weight support in pediatric rehabilitation. J Neurol Phys Ther. 2009;33(1):27-44. PMid:19265768 PMCid:PMC2982788. http://dx.doi.org/10.1097/ NPT.0b013e31819800e2

25. Mattern-Baxter J. Effects of partial body weight supported treadmill training on children with cerebral palsy. Pediatr Phys Ther 2009;21(1):12-22. PMid:19214072.

26. Woollacott MH, Burtner P. Neural and musculoskeletal contributions to the development of stance balance control in typical children and in children with cerebral palsy. Acta Paediatr Suppl. 1996;416:S58-62. PMid:8997450.

27. Nashner LM, Shumway-Cook A, Marin O. Stance posture control in select groups of children with cerebral palsy: deficits in sensory organization and muscular coordination. Exp Brain Res. 1983;49(3):393-409. PMid:6641837.

28. Dimitrijevic MR, Gerasimenko Y, Pinter MM. Evidence for a spinal central pattern generator in humans. Ann N Y Acad Sci. 1998;860:360-76. PMid:9928325

29. Barbeau H. Locomotor training in neurorehabilitation: emerging rehabilitation concepts. Neurorehabil Neural Repair. 2003; 17(1):3-11. PMid:12645440.

30. Mattern-Baxter K. Locomotor treadmill training for children with cerebral palsy. Orthopaedic Nursing. 2010; 29(3):169-73; quis 174-5. PMid:20505484.

\section{Correspondence}

Luanda André Collange Grecco

Universidade Nove de Julho

Av. Sebastião Henriques, 448, Vila Siqueira CEP 02723-050, São Paulo, SP, Brasil e-mail: luandacollange@ hotmail.com 